\title{
PBC: Better Solutions to Beat Feeling Beat
}

\author{
Naw April Phaw ${ }^{1}$. David E. J. Jones ${ }^{1}$
}

Published online: 4 March 2019

(c) Springer Science+Business Media, LLC, part of Springer Nature 2019

Primary biliary cholangitis (PBC) is a chronic cholestatic liver disease characterized by the destruction of the small intrahepatic bile ducts. The injury can be progressive, leading eventually to ductopenia at which point cholestasis is profound. PBC is a rare disease with an estimated prevalence of 35 per 100,000 of the population that predominantly affects females. Although the disease can be progressive, leading to biliary cirrhosis and the complications of endstage liver disease, there is also significant patient impact due to chronic symptoms that can be present throughout the disease course and are not restricted to patients with aggressive or advanced disease. Indeed, for many patients, symptoms represent the greatest clinical problem that they experience.

Although clinicians think of pruritus when considering the symptoms of $\mathrm{PBC}$, it is fatigue that this is the commonest symptom, affecting at least half of all patients. Approximately $20 \%$ of PBC patients report severe fatigue, often linked to significant social dysfunction [1]. The impact of this debilitating symptom contributes significantly to the poor quality-of-life (QoL) experienced by many patients with $\mathrm{PBC}$, which can be profoundly impaired [2], with tangible challenges including the ability to undertake the activities of everyday living, to sustain paid employment, and to undertake caring responsibilities (a particular challenge when patients frequently have young families). There is increasing evidence that disease impact on symptoms and QoL is the greatest in the youngest patients [3].

In the issue of Digestive Diseases and Sciences, Lee et al. present a timely and well-performed systematic review and meta-analysis that summarizes the current state-of-the-art regarding the treatment of fatigue in $\mathrm{PBC}$. The systematic

David E. J. Jones

David.Jones@ncl.ac.uk

Naw April Phaw

Naw-april.phaw@ncl.ac.uk

1 Institute of Cellular Medicine, Newcastle University, 4th Floor William Leech Building, Medical School, Framlington Place, Newcastle upon Tyne NE2 4HH, UK review identified 16 relevant studies: seven ursodeoxycholic acid (UDCA), the approved primary therapy; two liver transplant; two selective serotonin release inhibitors (SSRIs); one colchicine; one methotrexate; one cyclosporine; one mofadanil; and one obeticholic acid (OCA), approved second-line therapy for patients showing an inadequate response to UDCA, of which seven (five UDCA and two liver transplantation) were included in the meta-analysis. The conclusions make bleak reading for patients and clinicians, but perhaps at least provide important clarity and point the way ahead.

Conceptually, the two approaches to the treatment of fatigue in $\mathrm{PBC}$ are through superior treatment of the PBC disease process itself and through specific targeting of the symptoms and/or the mechanisms underlying them. In terms of understanding disease treatment, the meta-analysis clearly establishes that the current approved first-line therapeutic agent for PBC, UDCA, is not effective in the treatment of fatigue (albeit with an important caveat which will be highlighted later). The primary outcome of the study showed UDCA did not reduce the relative risk (RR) of the fatigue compared to the control group ( $\mathrm{RR}=0.86,95 \% \mathrm{CI}$ $0.69-1.08, p=0.19$ ). Furthermore, the randomized control trial of OCA as second-line therapy also showed no benefit in terms of fatigue. There is, therefore, no evidence to suggest that either first- or second-line drug therapy has any beneficial effect on PBC-related fatigue.

For patients with advanced liver disease, transplantation is, as is the case with most liver diseases, a highly effective intervention that significantly increases survival. The data relating to fatigue in the context of liver transplantation in $\mathrm{PBC}$, however, are complex and need to be interpreted with caution. Patients listed for transplantation experience very significant fatigue (in keeping with other advanced liver disease states and beyond that experienced by earlier stage PBC patients), with significant improvement following transplantation [4]. Importantly, transplanted patients do not return to normal fatigue levels; rather, they display fatigue levels similar to those experienced in the general PBC population [5]. Emerging UK data (from the UK-PBC consortium) suggest that the disease-specific disease symptoms and the 
nonspecific features of advanced cirrhosis are of additive impact on the QoL, with pre-transplantation patients experiencing both, with the latter, but not the former, resolving after transplantation. In simple terms, transplantation does not improve PBC-related fatigue, but does at least reverse the additional fatigue related to the presence of decompensated cirrhosis. Transplantation thus remains a treatment solely for advanced liver disease and should not be considered for the treatment of non-advanced but symptomatic disease. A corollary of this is that although effective treatment of the underlying disease with UDCA and/or OCA may not improve fatigue per se, it will at least reduce the impact of symptoms associated with advanced disease.

In terms of specific treatments for fatigue, the systematic review again failed to identify any effective therapies, with interventions such as modafinil, SSRIs, and rituximab considered ineffective [6]. A potential reason for this is the overall lack of understanding of the complex processes underlying fatigue in PBC. The pathogenesis of fatigue in PBC remains poorly understood but is likely to be multifactorial. There appears to be a central component to fatigue through possible inflammatory pathways linked to cholestasis. Even within central fatigue, there is evidence to suggest confounding and exacerbating processes such as sleep disturbance and autonomic dysfunction [7, 8]. To make matters worse, there appears also to be a peripheral component, including an element of metabolic dysfunction within skeletal muscle with an over-reliance on anaerobic metabolic pathways and the resulting development of profound muscular acidosis with exercise [9] that may be a secondary, deconditioning phenomenon. Whether it is a primary or a secondary phenomenon, it is likely to significantly contribute toward limiting the capacity of patients to recover from fatigue.

What can be done to improve this rather bleak picture? The review highlights a number of challenges that are encountered in the development and evaluation of treatments for fatigue in primary biliary cholangitis. First, the symptoms of the fatigue are broad and heterogeneous, with a complex interaction between central and peripheral components. Treatment that targets only one component in a patient with both might be ineffective if the peripheral muscular deconditioning component is secondary to central fatigue since even if the central component is reversed, the individual may be unable to overcome the peripheral deconditioning effects without specific support. Second, there is a lack of standardized tools to evaluate fatigue. In this meta-analysis, the measurement tools used for fatigue varied across the studies making it almost impossible to cross-compare studies and integrate the data. The PBC-40, a robust, patient-derived QoL measure with a fatigue domain, is the most commonly used tool, especially in trials conducted in recent years, and could have value as a trial standard [10]. Finally, if and when the mechanism of PBC-related fatigue is elucidated, novel targeted therapies will potentially be confounded by significant placebo effects, as reported in our recent rituximab trial [8]. Within these approaches, incremental small interventions such as addressing pruritus and its effect on sleep, autonomic dysfunction, and low-level depression, as well as exercise and lifestyle advice can have cumulative benefit. These approaches are an important part of holistic care but are very difficult indeed to address in clinical trials.

\section{Conclusions}

1. Since UDCA does not effectively treat fatigue, newer therapies are needed. Non-response of fatigue to UDCA just means that UDCA is not an effective treatment and should not be interpreted that the fatigue is not real or is not connected to PBC.

2. The existing and emergent second-line therapies [such as OCA] need to be formally evaluated for their actions on fatigue. The current "step-up treatment model" in which their use is restricted until after UDCA therapeutic failure may limit their effectiveness for fatigue.

3. A standard approach and symptom assessment "toolkit" [such as the PBC-40] is needed to facilitate integration of data sets between trials.

4. Improved understanding of the nature of central and peripheral fatigue is needed in order to appropriately stratify patients and target therapies in clinical trials.

5. Since deconditioning is an important peripheral component of fatigue, therapies should be aimed at physical conditioning in addition to pharmacologic treatments.

Although there has been considerable recent progress in the treatment of the $\mathrm{PBC}$ disease process, the fatigue element frustratingly remains in the dark, which always is the deepest just before the dawn.

\section{References}

1. Cauch-Dudek K, Abbey S, Stewart DE, Heathcote EJ. Fatigue in primary biliary cirrhosis. Gut. 1998;43:705-710.

2. Hale M, Newton JL, Jones DEJ. Fatigue in primary biliary cirrhosis. BMJ. 2012;345:e7004.

3. Dyson JK, Wilkinson N, Jopson L, Mells G, et al. UK-PBC consortium, the inter-relationship of symptom severity and quality of life in 2055 patients with primary biliary cholangitis. Aliment Pharmacol Ther. 2016;44:1039-1050.

4. Nevens F, Andreone P, Mazzella G, Strasser SI, et al. A placebocontrolled trial of obeticholic acid in primary biliary cholangitis. N Engl J Med. 2016;375:631-643.

5. Pells G, Mells GF, Carbone M, Newton JL, et al. The impact of liver transplantation on the phenotype of primary biliary cirrhosis patients in the UK-PBC Cohort. J Hepatol. 2013;59:67-73.

6. Lee JY, Danford CJ, et al. Treatment of fatigue in primary biliary 1 cholangitis: a systematic review and meta-analysis. Dig 
Dis Sci. (Epub ahead of print). https://doi.org/10.1007/s1062 0-019-5457-5.

7. Newton JL, Davidson A, Kerr S, Bhala N, et al. Autonomic dysfunction in primary biliary cirrhosis correlates with fatigue severity. Eur J Gastroenterol Hepatol. 2007;19:125-132.

8. Newton JL, Gibson GJ, Tomlinson M, Wilton K, et al. Fatigue in primary biliary cirrhosis is associated with excessive daytime somnolence. Hepatology. 2006;44:91-98.

9. Hollingsworth KG, Newton JL, Taylor R, McDonald C, et al. A pilot study of peripheral muscle function in primary biliary cirrhosis: potential implications for fatigue pathogenesis. Clin Gastroenterol Hepatol. 2008;6:1041-1048.
10. Jacoby A, Rannard A, Buck D, Bhala N, et al. Development, validation and evaluation of the PBC-40: a disease specific health related quality of life measure for primary biliary cirrhosis. Gut. 2005;54:1622-1629.

Publisher's Note Springer Nature remains neutral with regard to jurisdictional claims in published maps and institutional affiliations. 